\title{
Políticas de alfabetización digital. Educación e inclusión
}

\author{
Pini, Mónica
}

Resumen:

Este artículo se circunscribe al examen de fragmentos seleccionados de un documento de política educativa argentina. Se trata de la Res. CFE N ${ }^{\circ}$ 123/10 que aprueba el documento "Las Políticas de Inclusión Digital Educativa. El Programa Conectar Igualdad". Este texto tiene una pretensión de integralidad, ya que abarca los aspectos políticos y técnicos del uso de las tecnologías de la información y la comunicación (TIC) en las escuelas. En función del marco conceptual, el análisis crítico del texto se centra en la exploración tres ejes: la concepción de alfabetización, el papel adjudicado al Estado nacional y la inclusión como justicia curricular. El

Cuadernos del Centro de Estudios de Diseño y Comunicación №72

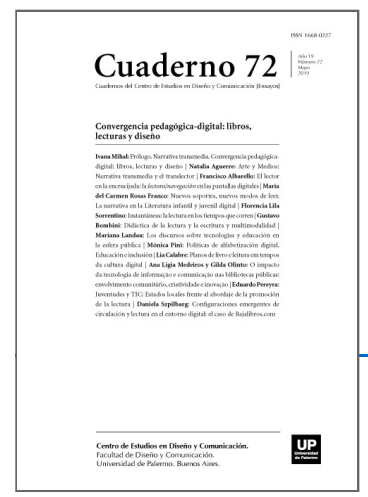

ISSN: 1668-0227

Convergencia

pedagógica-digital:

libros, lecturas y

diseño

Año XIX, Mayo 2019, Buenos Aires,

descargar PDF

ver índice de la publicación

Ver todos los libros de la publicación

compartir en Facebook

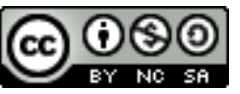

Esta obra está bajo una Licencia Creative Commons Atribución-NoComercialCompartirlgual 4.0 Internacional trabajo adopta un enfoque crítico, en una perspectiva relacional y sociocultural de la alfabetización (digital).

Palabras clave: Alfabetización digital - TIC - Estado - Inclusión.

$\left(^{*}\right)$ Doctora en Educación (University of New Mexico, USA), Magister en Administración Pública y Licenciada en Ciencias de la Educación (UBA). Directora del Centro de Estudios Interdisciplinarios en Educación, Cultura y Sociedad, Escuela de Humanidades, UNSAM. Dirige la Maestría en Educación, Lenguajes y Medios y es profesora regular de Educación, Cultura y Sociedad (UNSAM). Ha publicado trabajos sobre política e investigación educativa y análisis de discurso y educación.

Introducción

Este texto forma parte de un proyecto de investigación más amplio, cuyo objetivo1 es analizar qué concepciones de alfabetización digital se expresan en las políticas oficiales y de diferentes sectores involucrados en el período 2003-2015, en relación con la inclusión educativa. 
Durante las primeras décadas de este siglo, nuevos gobiernos populares en América Latina -el argentino entre ellos- marcan una nueva etapa caracterizada por:

- La recuperación de la política como dinámica de la vida ciudadana,

- La oposición al consenso de Washington y a la reforma de los noventa basada en el discurso único (neoliberal), y

- La recuperación de la centralidad del Estado en las políticas públicas (Feldfeber, 2010).

Estos importantes cambios permitieron hablar de un modelo de Estado "post-neoliberal". En los postulados y planes de gobierno desde el 2003 a 2015 en Argentina perdió lugar el discurso privatizador y gerencial que fue hegemónico en los noventa. Si bien diferentes consecuencias materiales y simbólicas persistieron, tanto en problemas sociales como en la naturalización de ciertos discursos, fue efectiva la ampliación de derechos sociales.

En efecto, en ese período, el entonces Ministerio de Educación de la Nación imprimió un rumbo dinámico y dio prioridad a la concreción de políticas y programas destinados a combatir la desigualdad. Al mismo tiempo, las políticas generales con respecto al empleo, al salario y a la protección social contribuyeron en gran medida al mejoramiento de los horizontes educacionales. La asignación universal por hijo (AUH)2 , por ejemplo, contribuyó a que la obligatoriedad de la escuela secundaria establecida en la Ley de Educación Nacional se haga posible para los sectores de menores recursos (Pini, 2011).

\section{Aspectos teórico metodológicos}

El estudio es de carácter cualitativo e incluye una descripción y análisis documental contextualizado. La perspectiva adoptada es la del análisis crítico de discurso (ACD), combinada con análisis político, la sociología y la pedagogía críticas. Este artículo se circunscribe al examen de un documento de política educativa argentina, en función de su relevancia para el tema que nos ocupa y en el marco de la normativa previa.

Los discursos legales y políticos son textos que tienen carácter formal y suponen una preparación y revisión por más de una persona. Carecen de marcas de autor, lo cual crea un efecto de verdad y objetividad, y presuponen un contexto previo conocido por los lectores (Calsamiglia y Tuson, 2007). A diferencia de otros textos, tienen una intención retórica explícita, ya que buscan generar adhesión o cumplimiento y modificar en algún sentido las prácticas sociales, lo cual no significa que se hagan explícitas todas las implicaciones ideológicas.

El trabajo adopta un enfoque crítico, en una perspectiva relacional. En la línea de los trabajos de Buckingham (2008), Da Porta (2011) y Selwyn (2014), se considera que para pensar la tecnología en educación, lo primero que hay que pensar es la educación, y discutir el supuesto determinista de que las tecnologías poseen cualidades inherentes y que por lo tanto son capaces de tener efectos o impactos particulares predeterminados y predecibles sobre los estudiantes, los docentes y toda la sociedad.

La perspectiva sociocultural de la alfabetización (digital), que en América Latina -y gran parte de Occidenteabreva en los trabajos Paulo Freire, se encuentra en consonancia con la tradición de los estudios sobre New Literacy, New Literacies, Digital Literacies, Media Education, Critical Discourse Analysis, Critical Literacy, etc. 
(Rogers, 2003; Landau, 2008; Rodríguez Illera, 2004; Lankshear y Knobel, 2008; Albarello, 2011; Ferreiro, 2013; Ferrés, 2000, 2008; Orozco Gómez, 2001, 2010; Buckingham, 2008). Desde esta perspectiva, se concibe la alfabetización digital como un conjunto de prácticas sociales que fundamentalmente implican la capacidad de leer y escribir comprensivamente y usar información en múltiples formatos y soportes, con diferentes propósitos y en contextos específicos (Lankshear y Knobel, 2008). Esta conceptualización se aparta de la idea de una educación informática o basada en habilidades técnicas u operativas. Al mismo tiempo permite pensar integralmente en un estudiante sujeto de derecho, que necesita de las enseñanzas escolares en tanto estas reconozcan sus intereses y potencien sus posibilidades.

En función de esta definición de alfabetización digital se han rastreado ya estos significados en la Ley de Educación Nacional (LEN) y en la Ley de Servicios de Comunicación Audiovisual (LSCA), en tanto dos de las normativas más relevantes de este siglo referidas a temas de educación, tecnologías digitales y medios (Mihal y Pini, 2016). En ellas se ha se- ñalado, primeramente, la responsabilidad principal del estado en garantizar los derechos a la comunicación y la educación. Asimismo se han identificado los elementos más importantes destinados a facilitar y promover la alfabetización digital de manera inclusiva, de acuerdo con nuestra definición, aunque no se utiliza esa denominación.

En este trabajo se analiza otra de las principales normativas de nivel nacional, y al mismo tiempo federal, relacionada con el tema, en particular una de las resoluciones fundamentales con respecto a la alfabetización digital, emanada del Consejo Federal de Educación (CFE), conformado por todos los ministros de educación de las provincias argentinas y la Ciudad Autónoma de Buenos Aires3. Se trata de la Resolución CFE N 123/10, que aprueba el documento "Las Políticas de Inclusión Digital Educativa. El Programa Conectar Igualdad". Su texto tiene una pretensión de integralidad, ya que abarca los aspectos políticos y técnicos del uso de las TIC en las escuelas.

En función del marco conceptual, el análisis crítico del texto se centra en la exploración de tres ejes: la concepción de alfabetización digital, el papel adjudicado al estado nacional y la inclusión como justicia curricular en un marco político pedagógico.

\section{Antecedentes inmediatos}

En el contexto planteado más arriba, el Ministerio de Educación (2003-2015) dio prioridad a la concreción de políticas y programas destinados a combatir la desigualdad. Si bien la Ley de Educación Nacional (LEN N ${ }^{\circ}$ 26206/2006) afirma la responsabilidad del Estado de garantizar el derecho a la educación, establecido en la Constitución Nacional, la ausencia de prioridad de la educación pública en la agenda gubernamental en las últimas décadas del siglo XX había beneficiado a la educación privada, y reducido la pública prácticamente a la atención de los sectores más desfavorecidos.

Dentro del período de gobierno en el que nos situamos para el análisis, el año 2010 representa un hito en lo que se refiere a las iniciativas de la LEN con respecto a la introducción de las tecnologías de la información y la comunicación (TIC) en la escuela secundaria. Si bien ya en 2008 -en el marco de la Ley de Educación Técnico Profesional N²6.058/05- se había aprobado el Programa Nacional de Equipamiento Informático para las Instituciones de Educación Técnico Profesional que dictaran carreras técnicas de nivel secundario (RCFE N50), y en 2009 se establecía el Programa Nacional "Una Computadora para Cada Alumno", para ser implementado 
en escuelas técnicas de gestión estatal dependientes de las provincias y de la Ciudad Autónoma de Buenos Aires, ambos programas estaban destinados solamente a las escuelas de educación técnica.

En 2010 se crea el "Programa Conectar Igualdad. Com. Ar" "de incorporación de la nueva tecnología para el aprendizaje de alumnos y docentes" (PCl, Decreto $\mathrm{N}^{\circ}$ 459/10)4 . En los considerandos se mencionan los tratados internacionales relacionados con el derecho a la educación, así como la Ley de Educación Nacional, en particular en lo referido a los objetivos de la educación secundaria y de la educación especial. Se reafirma la responsabilidad del Estado en garantizar y mejorar la educación, no sólo como enunciado sino mediante la asignación de los recursos necesarios. En este sentido, alude a la Asignación Universal por Hijo (AUH) por su incidencia en el aumento de la matrícula del nivel secundario y en la disminución de la pobreza y la indigencia, y considera que resulta una oportunidad histórica para promover la inclusión digital y capacitar a los docentes para esto. Es así que se crea un Comité Ejecutivo interministerial5 presidido por el ANSES y un programa presupuestario específico para el $\mathrm{PCl}$, destinado a estudiantes de escuelas públicas de todo el país.

Llama la atención que colocaran el dominio .com en el título de creación, como si se hubiera pensado en que lo maneje una empresa, cuando el programa pertenecía al sector público y correspondía al dominio .gob, en el que luego estuvo siempre, con el nombre Conectar Igualdad.

A partir del PCl, se decide subsumir el Programa Nacional "Una Computadora para Cada Alumno" de las escuelas técnicas como parte del "Programa Conectar Igualdad" (RCFE N¹14/10), y se aprueba el documento: "Las políticas de inclusión digital educativa. El programa Conectar Igualdad", en el que se acuerdan las líneas prioritarias para una política de integración de TIC (RCFE N¹23/10), en consonancia con la LEN, en particular su título VII.

\section{Lineamientos}

El documento "Las políticas de inclusión digital educativa. El programa Conectar Igualdad" es extenso y se compone de 4 partes: 1. Presentación, 2. Fundamentación, 3. Antecedentes, y 4. El Programa Conectar Igualdad ( $\mathrm{PCl})$. En este artículo, por razones de extensión, nos centramos en las dos primeras.

Como se dijo más arriba, el enfoque se plantea como integral, referido tanto a lo político educativo como a lo técnico. La fundamentación incluye los principios orientadores de la política federal referida a las tecnologías de la información y la comunicación (TIC) y las líneas estratégicas de una política de educación y TIC.

Los antecedentes se refieren a iniciativas previas de nivel nacional y provincial. En el ámbito nacional, se destaca el desarrollo de Educ.ar y del Canal Encuentro. Con respecto a las experiencias de modelo de laboratorio se mencionan el Programa de Mejoramiento de la Enseñanza Media (PRODYMES II) y el Plan Social Educativo (PSE) desarrollados en los '90; el programa RedEs (1998-1999); el Programa de Mejoramiento de la Enseñanza Media II (PROMSE); el Programa de Apoyo a la Política de Mejoramiento de la Equidad Educativa (PROMEDU); el Proyecto de Mejoramiento de la Educación Rural (PROMER); el Programa Integral para la Igualdad Educativa (PIIE); y el FOPIIE, Programa para el Fortalecimiento Pedagógico de las Escuelas beneficiarias del PIIE (2003-2009). Desde el Instituto Nacional de Formación Docente, se implementaba una línea de acción para la incorporación de las TIC en la Formación, con la instalación campos virtuales en cada IFD. 
En cuanto a las experiencias basadas en el modelo 1 a 1 y aulas digitales, además de algunas experiencias piloto, el antecedente directo es el Programa Nacional del INET-ME “Una computadora para cada alumno" (Res. CFE N 82/09), implementado en escuelas técnicas públicas de gestión estatal de todo el país. En el ámbito provincial se menciona programas basados en ese modelo en San Luis, Río Negro, Ciudad de Buenos Aires y La Rioja.

El apartado sobre el PCI detalla -con cierto grado de redundancia-, finalidades, objetivos, el enfoque pedagógico, la estrategia educativa, la adquisición de equipamiento y sus modalidades según los destinatarios, la gestión, el seguimiento y la evaluación del programa. Además de situarse en el marco de la LEN, en particular de su Título VII, el documento menciona múltiples acciones desplegadas tanto por los estados provinciales como por el estado nacional, tales como distribución de equipamiento, el portal Educ.ar y el Canal Encuentro, líneas focalizadas de capacitación docente, programas y proyectos abocados a la incorporación de la temática TIC en las prácticas educativas.

El documento expresa como propósito general "definir lineamientos políticos y técnicos para la incorporación, integración y aprovechamiento pedagógico de las TIC en el sistema educativo" (p. 4). Una de las primeras afirmaciones es que "la integración de las TIC en el ámbito de las instituciones educativas implica repensar tanto la configuración institucional como las prácticas que de ella derivan, el desarrollo curricular-escolar, así como el trabajo de docentes y alumnos en las aulas, orientados a integrar estas herramientas en la formación de ciudadanos críticos, creativos y responsables, así como de nuevos perfiles formativos para el mundo del trabajo". Asimismo ubica estas políticas en el marco del Plan Nacional de Educación Obligatoria6 y del Plan Nacional de Formación Docente7 que definían metas de igualdad e inclusión, calidad educativa, y fortalecimiento institucional. Por un lado se define un encuadre político-pedagógico y por otro se expresa la idea de que se requiere repensar todas las dimensiones del hecho educativo, así como tener presentes los objetivos de la formación a la cual se integrarían las TIC. Al mismo tiempo, entiende la educación como un derecho humano fundamental que los estados "tienen la obligación de promover con el fin de asegurar la igualdad de oportunidades para toda la población." Además de la obligación de promover el derecho a la educación, postula garantizarlo, tal como lo expresa la LEN en su art. 2, y al mismo tiempo se obliga a asegurar la igualdad de oportunidades. Seguidamente se refiere a los art. 7 y 8 de la LEN en los que "el Estado garantiza el acceso de todos/as a la información y al conocimiento", como "responsable de garantizar equidad en el acceso a las TIC, para favorecer la circulación y producción de conocimiento y la inclusión social, cultural y educativa" (Res. $\mathrm{N}^{\circ}$ 123, p. 6). Con este fin, destaca la importancia de las TIC "al servicio de las prácticas pedagógicas cotidianas, imprimiéndoles un sentido innovador".

Es claro que se requiere renovar las prácticas escolares, si bien la idea de "innovación" marca el discurso dominante y el sentido común alrededor de las TIC, como sinónimo de inherente mejoramiento en la enseñanza y para diferenciar esta era digital de las anteriores pre-digitales. Es una expresión que en el discurso social asocia indisolublemente todo lo "nuevo" que trae la tecnología digital con bueno y mejor, en términos de eficiencia y efectividad en los aprendizajes, más allá de las circunstancias. Por lo general el uso de estos términos desestima todas las múltiples y diferentes situaciones que se producen en contextos de TIC en el aula, así como la necesidad de revisar la concepción pedagógica que le da marco a las prácticas con tecnologías (Selwyn, 2013). Al mismo tiempo, enmascara el enorme campo de intereses comerciales detrás de estas propuestas, la brecha social digital entre los jóvenes, y que no hay dispositivos únicos que solucionen el problema de la educación. 
Aparentemente éste no sería el caso, ya que a lo largo del texto se reitera el énfasis en lo nuevo, ligado a los saberes, la comprensión y el aprendizaje:

El ingreso de las TIC a la escuela se vincula con la exigencia de nuevos saberes, la respuesta a ciertas demandas del mundo del trabajo y la necesidad de comprender y participar en una realidad mediatizada. El abordaje y formación sistemática sobre TIC resulta una oportunidad para que niños/as, jóvenes y adultos/as puedan desarrollar saberes y habilidades específicos que estén puestos al servicio del desarrollo de los contenidos curriculares (p. 7).

Si bien, como afirma Da Porta $(2011,2015)$, socialmente se desarrolla la necesidad no cuestionada de la incorporación de las tecnologías en la escuela, por otra parte esta norma intenta condicionarla a la multiplicidad de factores que inciden en los contextos de aprendizaje.

La Resolución toma como principios rectores una serie de criterios que se desprenden de la Ley de Educación Nacional, y que son retomados por el Plan Nacional de Educación Obligatoria:

- La equidad y la inclusión

- La calidad

- La formación de la ciudadanía

- La innovación

Con respecto a la equidad y la inclusión, destaca el problema de la desigualdad de oportunidades no sólo de carácter económico, sino político, pedagógico y cultural. Reconoce que se priorizaron las políticas para posibilitar el acceso a los sectores más desfavorecidos, asignando recursos para ese fin. En ese sentido, señala que las políticas han tenido un sesgo claramente instrumental y que se requiere impulsar la integración pedagógica significativa.

En cuanto a la calidad, siempre teniendo como marco la LEN, postula que la integración de TIC aportará a la buena calidad educativa si se adopta "una perspectiva multidimensional que contemple sus potencialidades para la actualización curricular, la construcción de conocimiento y la apropiación de diversos lenguajes para la expresión" (p. 8). Agrega la necesidad de procesos formativos que incluyan lecturas críticas, participación y aprendizaje colaborativo con producciones en diversos soportes y la necesidad de diálogo entre generaciones. En el mismo apartado se refiere a la "modernización" (comillas en el original) de las prácticas en función de los objetivos de política educativa. Despierta interrogantes este uso del término encomillado, ya que justamente es un término poco usado en educación y en general, cuestionado por las sucesivas crisis de la modernidad capitalista. Parece apuntar a una renovación o a la ya mencionada innovación, que constituye el cuarto criterio de los que menciona.

La formación de ciudadanía hace referencia a poner en juego la dimensión ética de los procesos formativos, y preparar a los estudiantes como sujetos conscientes y ciudadanos con prácticas democráticas. 
Para referirse a la innovación, afirma que "Abordar las TIC desde la perspectiva de la innovación pedagógica permite reposicionar a los docentes desde la experiencia de su conocimiento didáctico" (p. 10). Se trata de partir de los saberes propios del docente, incorporando el dominio operativo de la tecnología como primer paso para desarrollar proyectos que incorporen todo su potencial. Innovación es explorar, experimentar, desarrollar capacidades para producir cambios en las prácticas pedagógicas:

Cambios de actitudes, pensamientos, culturas, contenidos, modelos de trabajo y sentidos... generar programas y proyectos que inciden sobre los aspectos curriculares, las estrategias de enseñanza y de aprendizaje, los modelos didácticos, las dinámicas del aula y las formas de organización institucional. (pp. 10-11).

Estos cambios en la organización institucional dependen en gran medida de decisiones políticas generales, pero la amplitud de expectativas que se depositan en la innovación parece estar asentada en las "10 líneas estratégicas fundamentales" que se proponen luego para asegurar una política integral de TIC para el sistema:

1. Producción, acceso y actualización de contenidos y/o software.

2. Incorporación de las TIC en los contenidos curriculares.

3. Calidad de las prácticas pedagógicas y usos educativos de las TIC.

4. Desarrollo profesional docente.

5. Cambios organizacionales en las instituciones educativas y nuevos roles para el trabajo con TIC.

6. Provisión, instalación, mantenimiento y actualización de equipamiento, software y redes.

7. Sistematización de información y experiencias e Investigación.

8. Gestión de la Información.

9. Fortalecimiento de los equipos técnicos y referentes TIC de los Ministerios de Educación.

10. Articulación entre Estado, Universidades, organizaciones de la sociedad civil y el sector privado.

Estas líneas corresponden a los aspectos materiales y simbólicos, curriculares, pedagógicos, políticos, organizacionales, formativos, metodológicos, mediaciones y soportes orientados a asegurar la buscada integralidad de la política.

La concepción de alfabetización digital

A lo largo del extenso texto de la resolución, se percibe una oscilación entre los significados más instrumentales y que enfatizan el poder transformador de las TIC en sí mismas, y los que enfatizan su significado político pedagógico y cultural.

Por ejemplo, se busca "disminuir las brechas entre los que tienen acceso y los que no, contribuyendo a mejorar los indicadores de alfabetización digital de la población y el desarrollo de nuestro país" (p. 4). En esta acepción 
la alfabetización digital tiene un significado más de acceso operativo cuantificable que de uso crítico de la tecnología.

Al mismo tiempo describe un abordaje pedagógico y cultural de las TIC como contenidos curriculares, desarrollo de habilidades analíticas, cognitivas, creativas y comunicativas de alumnos, docentes y directivos, y a su vez un enfoque político pedagógico (p. 25), con el objetivo de actualizar los soportes y recursos pedagógicos y democratizar el acceso a la información y al conocimiento. El abordaje de las TIC constituye una estrategia de carácter transversal y una herramienta de trabajo pertinente que responde a las especificidades de las disciplinas y abre la posibilidad de vincularlas con el mundo académico, político, social, del trabajo y de la cultura.

Por otra parte, afirma el papel de la escuela pública valorizando "la incorporación de las TIC como una herramienta tecnológica potente en su capacidad de modificar y acompañar los procesos de enseñanza de los docentes y de los aprendizajes de los alumnos" (p. 22). En estas definiciones se encuentra cierta ambivalencia que tanto se podría leer como contradictoria o como albergando una amplitud que abarca varios de los significados circulantes. Si en lugar de considerarlos opuestos los vemos como un continuo que va desde el acceso al artefacto, ligado a una cuestión de justicia distributiva, hasta el enfoque pedagógico y cultural que llega a la lectura crítica de las TIC y los medios como objeto de estudio, pasando por la identificación de la tecnología como un elemento de la innovación en la educación, esto nos obliga a profundizar la reflexión y el debate en el marco de la mayor democratización e inclusión educativa.

\section{El papel adjudicado al estado nacional}

Como se afirmó en la introducción, en el período 2003-2015 se produce una revalorización de la política, en particular de las políticas desde el Estado, estableciendo nuevos marcos legales que le dieron sustento. El PCI fue una de las políticas educativas que constituyeron los procesos de restitución del Estado, de lo público y del sujeto político (Huergo, 2011). En ella confluyeron el resto de las iniciativas y programas, centralizando la política estatal en torno a las TIC (Llimás et al., 2011), y una de sus expresiones más importantes es la Res. №123/10. Las líneas estratégicas ubican al estado nacional como responsable sin dejar de lado el papel de los estados provinciales y los mecanismos federales. En particular la última, establece: "Propiciar la articulación entre el sector público y el sector privado, la Universidad y la empresa, los organismos descentralizados y las organizaciones de la sociedad civil para un mejor aprovechamiento de recursos, experticias, infraestructura y acceso a fuentes de financiación."

El sentido de este tipo de articulaciones está orientado por el Estado en función de las políticas públicas, ya que de otro modo prevalece la lógica empresarial del beneficio privado. Este aspecto no se menciona en el texto, pero si bien varias empresas tuvieron participación en la provisión de diferentes insumos, el proyecto político general era orientado y conducido por un Estado presente.

La inclusión como justicia curricular en un marco político pedagógico

EI PCI se proponía mejorar la enseñanza y el aprendizaje a la vez que llegar con las TIC a toda la comunidad, a través de una netbook que cada estudiante -de educación pública secundaria, especial y formación docente- 
llevaba a su casa, promoviendo efectos educativos tanto en la escuela como en el entorno familiar, en particular para los que no tenían acceso previo a una computadora.

Desde sus fundamentos, Conectar Igualdad se plantea como una estrategia para fortalecer y revalorizar la escuela pública, pero también para contribuir a la inclusión social a través de la reducción de la brecha digital y la democratización del acceso a la circulación y producción de conocimientos. (Llimás et al., 2011, p. 69)

Esto se profundiza en el texto de la Res. $N^{\circ}$ 123/10. Las formas de inclusión que plantea se refieren a:

1) Promover nuevos procesos de aprendizaje en alumnos con diferentes discapacidades y fortalecer procesos de integración a partir de la utilización de las TIC en su formación escolar y laboral de las escuelas especiales; y

2) Mejorar la calidad de los procesos de enseñanza y de aprendizaje, a partir de integrar el uso de las TIC en las prácticas pedagógicas y el trabajo con las áreas de conocimiento, tanto por parte de docentes como de alumnos que impacten positivamente en sus trayectorias educativas (p. 25).

Con este fin desarrolla un enfoque pedagógico en el que se detallan "algunos de los procesos que requieren motorizarse para convertir al equipamiento y los contenidos digitales en entornos significativos de aprendizaje", con el extraordinario aporte de Educ.ar y del Canal Encuentro.

Si bien se ha criticado que las netbooks contienen los programas más usados de la compañía Microsoft, como política universal de inclusión educativa el PCl impulsó, además de la producción de contenidos audiovisuales propios de acceso público, el desarrollo autónomo de software libre por el PNIDE-ME, que se incluye en las computadoras con el nombre de Huayra.

Reflexiones finales

La resolución que analizamos aquí abre posibilidades, en consonancia con la Ley Nacional de Educación, de procesos político pedagógicos críticos alrededor del uso de las tecnologías en la enseñanza que están en consonancia con las definiciones integrales que adoptamos de alfabetización digital.

Estos procesos, que abarcan -y no se agotan en- prácticas áulicas con diferentes recursos textuales, visuales y sonoros que reflejan la convergencia pedagógico-digital: libros, lecturas, diseño e imagen, pueden conducir a que Ixs jóvenes realicen una apropiación creativa y transformadora de la tecnología que los acerque a un aprendizaje sistemático y significativo de diferentes contenidos. Lo fundamental en dicha alfabetización no son los dispositivos en los que se lee sino las prácticas pedagógicas que posibilitan la construcción crítica de la lectura en cualquier soporte, base de la democratización del saber y de la comunicación.

La alfabetización digital para la ciudadanía implica aprender a leer/descifrar tanto un noticiero de televisión como un texto literario. Para esas lecturas se requiere adquirir herramientas para comprender, al mismo tiempo que discernir, valorar y escoger en qué textos y a través de qué estrategias se apunta a fortalecer los estereotipos o a revisar las concepciones que tenemos de la política y de la familia, de la cultura y de la sexualidad (Pini, Amaré, Cerdeiro y Terzian, 2017) 
Sin duda la norma no desconoce el ambiente hegemónico de celebración e instrumentalización de las TIC e intenta incorporar la multiplicidad de aspectos relacionados, en un intento de minimizar las tensiones y contradicciones internas tanto de la política como de las mediaciones en su efectivización. Sin embargo, las tensiones existen y conviven significados establecidos por el pensamiento dominante con la gestación de posibilidades redistributivas, tanto de bienes como de conocimiento y reconocimiento.

Tal como se ha expresado, estas normativas surgen en un contexto político que pone al Estado y la cuestión social en el centro de la escena, enfatizándose la democratización en el acceso a bienes y servicios simbólicos, la perspectiva de derechos, de diversidad cultural y de formación integral de la ciudadanía (Pini y Mihal, 2016). Tanto las definiciones políticas como el lugar en el que se colocaba el estado se orientaban a procurar y asegurar posibilidades de inclusión de los estudiantes en tanto se alinea con criterios de justicia curricular en un marco político pedagógico. En este marco, lo nuevo y la innovación no se glorifican aparte de su contenido, sino que se insertan en revisiones más sustantivas de las prácticas.

Los cambios en lo que se refiere a educación en la etapa post 2015 están ligados más a las políticas generales que a modificaciones legislativas, si bien las que sí hubo muestran una tendencia clara a la liberalización y a la reducción de derechos sociales y laborales. A partir del cambio de gobierno en 2015, en que el estado vuelve a adoptar políticas neoliberales aún más drásticas que en los noventa sin modificar gran parte de la normativa vigente, vuelve a ponerse en valor la importancia del proyecto político y del modelo de estado que da marco y posibilidades de concreción y cumplimiento pleno de las normas.

Notas

1. PICT 2015-0373: "Innovación en políticas educativas: discursos y estrategias en torno a la alfabetización digital en el marco de la inclusión", Plan Argentina Innovadora 2020, FONCYT. Financiamiento por Resolución Nº 240-16 ANPCYT. El estudio es de carácter cualitativo e incluye una descripción y análisis documental contextualizado. La perspectiva adoptada es la del análisis crítico de discurso (ACD), combinada con análisis político, la sociología y la pedagogía críticas.

2. Dto. $1602 / 2009$, un monto mensual que abona el Estado a los jefes/jefas de hogar por cada hijo, con el requerimiento de que Ixs niñxs asistan a la escuela y concurran a realizarse control de salud y las vacunas obligatorias. Lo nuevo de esta medida es que no exige a los padres tener un trabajo legal, sino que se encuentra asociado con la edad de Ixs niñxs, alcanzando a desempleados, trabajadores informales y domésticos: los sectores sociales más vulnerables. Desde el comienzo de la implementación del programa, el nivel de indigencia y la pobreza disminuyeron, así como los niveles de desigualdad. Se incorporaron a las escuelas 250.000 nuevos estudiantes, especialmente en el nivel secundario (Cufré, 2010; Lukin, 2010).

3. El Consejo Federal de Educación es el organismo de concertación, acuerdo y coordinación de la política educativa nacional para asegurar la unidad y articulación del Sistema Educativo Nacional. Su presidente es el Ministro de Educación de la Nación y se encuentra a su vez integrado por la máxima autoridad educativa de cada jurisdicción y tres representantes del Consejo de Universidades. Las Resoluciones del Consejo son de carácter obligatorio conforme lo establece la Ley de Educación Nacional y el Reglamento de Funcionamiento del mismo, aprobado por Resolución CFE № 1/07 (http://portal.educacion. gov.ar/consejo/2009/12/04/el-consejo/) 
4. "Con el fin de proporcionar una computadora a alumnas, alumnos y docentes de educación secundaria de escuelas públicas, de educación especial y de institutos de formación docente, capacitar a los docentes en el uso de dicha herramienta y elaborar propuestas educativas con el objeto de favorecer la incorporación de las mismas en los procesos de enseñanza y aprendizaje".

5. Jefatura de Gabinete de Ministros, Ministerio de Educación y Ministerio de Planificación Federal, Inversión Pública y Servicios.

6. ME, 2009, posteriormente se fue actualizando.

7. ME, 2008, posteriormente se fue actualizando.

Bibliografía

Albarello, F. (2011). Leer/navegar en Internet. Las formas de lectura en la computadora. Buenos Aires: La Crujía.

Buckingham, D. (2008). Más allá de la tecnología. Aprendizaje infantil en la era digital. Buenos Aires: Manantial.

Calsamiglia Blancafort, H. y Tuson Valls, A. (2007). Las cosas del decir. Manual de análisis del discurso. Barcelona: Ariel Lingüística.

Cufré, D. (2008). "Línea de partida para combatir la pobreza”, Diario Página 12, acceso el 10/7/10. Disponible en: http://www.pagina12.com.ar/diario/economia/2-145350 -2010-05-08.html

Da Porta, E. (comp.) (2011). Comunicación y educación; debates actuales desde un campo estratégico. Córdoba: UNC.

Da Porta, E. (comp.) (2015). Las significaciones de las TIC en educación: políticas, proyectos, prácticas. Córdoba: Ferreyra Editor.

Feldfeber, M. (2010). "De la profesionalización al desarrollo profesional. Algunas notas para pensar las políticas de formación docente". En Dalila Oliveira y Feldfeber Myriam, Nuevas regulaciones educativas en América Latina. Políticas y procesos del trabajo docente. Lima, Perú: Asociación civil Universidad de Ciencias y Humanidades, fondo Editorial.

Ferreiro, E. (2013). El ingreso a la escritura y a las culturas de lo escrito. México: Siglo XXI.

Ferrés, J. (2000). Educar en una cultura del espectáculo. Barcelona-Buenos Aires: Paidós.

Ferrés, J. (2008) La educación como industria del deseo. Un nuevo estilo comunicativo. Barcelona: Gedisa.

Huergo, J. (2011). "Sentidos estratégicos de comunicación/educación en tiempos de restitución del Estado". En Da Porta (comp.) Comunicación y educación; debates actuales desde un campo estratégico, pp. 15-39.

Córdoba: UNC. 
Landau, M. (2008). "Los docentes en los discursos sobre alfabetización digital”. Razón y Palabra, n 63, Medios informáticos en la formación de profesores, julio-agosto.

Lankshear, C. y Knobel, M. (2008). "Introduction.Digital literacies-Concepts, policies and practices”. En Lankshear, C. \& Knobel, M. (editors) Digital Literacies. Concepts, policies and practices, pp. 1-16. NY: Peter Lang.

Llimós, G., Hamada, P., Palmero, M. y Yeremian, G. (2011). “TIC, políticas educativas y mercado: reflexiones desde y hacia el campo comunicación y educación". En Da Porta (comp.) Comunicación y educación; debates actuales desde un campo estratégico, pp. 60- 92. Córdoba: UNC.

Lukin, T. (2010). "Vamos a trabajar para que no se vuelvan a ir”, Diario Página 12. Acceso el 10/10/2010Disponible en: http://www.pagina12.com.ar/diario/economia/2-149201-2010-07-10.html

Mihal, I. y Pini, M. E. (2016). “Acerca de la alfabetización digital en normativas nacionales de Argentina”. En W. Iriondo, I. Bras Ruiz, P. Mussi Escobar y M. C. Gamboa M. (Eds.) Utilizando tecnologías en la educación para fortalecer la práctica docente en América Latina. Revisiones teóricas - Experiencias prácticas. Bogotá: IDEAUSaCh, RILET, y UNAD-Colombia.

Orozco Gómez, G. (2001). “Audiencias, televisión y educación: una deconstrucción pedagógica de la televidencia y sus mediaciones". Revista Iberoamericana de Educación n² 27, pp. 155-175.

Orozco Gómez, G. (2010). Niños, maestros y pantallas. Observatorios de televisión en la escuela. México: COECYTJAL-UDG.

Pini, M. E. (2011). “¿Post neoliberalismo? Políticas educativas recientes en Argentina” en A. Montané y J. Beltrán (coords.) Miradas en movimiento. Textos y contextos de políticas de educación. Valencia: Editorial Germania.

Pini, M. E. y Mihal, I. (2016). "Alfabetización digital y política educativa democrática en dos normativas de Argentina". Revista Diálogos de la Comunicación, edición no. 93, "Comunicación y Educación en la era digital".

Pini, M. E.; Amaré, M.; Cerdeiro, C. y Terzian, C. (2017). "Mundo escolar y mundo juvenil. La escuela frente al desafío de derribar los muros". En Pini, M.E., Landau, M. y Valente E. (comp.) Tecnologías para el aula: análisis y propuestas pedagógicas, pp. 17-42. Buenos Aires: Aique.

Rodriguez Illera, J. L. (2004). Las alfabetizaciones digitales- En Bordón. Revista de Pedagogía, Vol. 56, № 3-4, pp. 431-441.

Rogers, R. (2003). A critical discourse analysis of family literacy practices: Power in and out of print. Mahwah, $\mathrm{NJ}$ : Lawrence Erlbaum.

Selwyn, N. (2014). Distrusting educational technology. Critical questions for changing times. NY: Routledge. 
Abstract: This article is limited to the examination of selected fragments of an Argentine educational policy document. This is Res. CFE N¹23/10 that approves the document "The Policies of Digital Educational Inclusion. The Connect Equality Program". This text has a pretension of integrality, since it covers the political and technical aspects of the use of ICT in schools. Depending on the conceptual framework, the critical analysis of the text focuses on the exploration of three axes: the conception of literacy, the role assigned to the national state and inclusion as curricular justice. The work adopts a critical approach, from a relational and sociocultural perspective of (digital) literacy.

Key words: Digital Literacy - ICT - State - Inclusion.

Resumo: Este artigo está limitado ao exame de fragmentos selecionados de um documento da política educativa argentina. Este é o Res. CFE n. ${ }^{\circ}$ 123/10 aprovando o documento "As políticas de inclusão educacional digital". O Programa Conectar Igualdad". Este texto tem uma pretensão de integralidade, uma vez que abrange os aspectos políticos e técnicos do uso das tecnologias de informação e comunicação (TIC) nas escolas. Dependendo da estrutura conceitual, o análise crítica do texto centra-se na exploração de três eixos: a concepção de alfabetização, o papel atribuído ao Estado nacional e inclusão como justiça curricular. O trabalho adota uma abordagem crítica, de uma perspectiva alfabetização relacional e sociocultural (digital).

Palavras chave: Alfabetização digital - TIC - Estado - Inclusão.

[Las traducciones de los abstracts al inglés y portugués fueron supervisadas por el autor de cada artículo]

Políticas de alfabetización digital. Educación e inclusión fue publicado de la página 95 a página107 en Cuadernos del Centro de Estudios de Diseño y Comunicación N72 